\title{
Parâmetros de duração dos sinais acústicos da deglutição de indivíduos sem queixa
}

\author{
Duration parameters of swallowing acoustic signals in subjects \\ without complaints
}

\author{
Olívia Helena Gomes Patatas ${ }^{1}$, Maria Inês Rebelo Gonçalves², Brasília Maria Chiari², Ingrid Gielow ${ }^{3}$
}

\begin{abstract}
RESUMO
Objetivo: Investigar a consistência da duração e a reprodutibilidade do padrão dos eventos acústicos relacionados à deglutição de indivíduos sem queixa. Métodos: Oitenta e oito mulheres e 76 homens, entre 6 e 85 anos, com um microfone acoplado ao pescoço e ligado a um computador, realizaram nove deglutições de saliva, e de $5 \mathrm{ml}$ e $10 \mathrm{ml}$ de água. A partir do registro acústico dos sons captados, analisaram-se as durações dos intervalos entre os picos dos dois principais cliques de deglutição (T), da apneia da deglutição (dA), e a relação entre ambos (T/dA). Resultados: Não houve diferenças quanto às durações médias dos tempos entre os picos dos dois cliques da deglutição (T), da apneia da deglutição (dA) e da relação T/dA nas diferentes faixas etárias ou entre os gêneros, com exceção do índice T/dA para as deglutições de $5 \mathrm{ml}$ de água. Conclusão: Os sons da deglutição apresentam um padrão característico e reprodutível, sendo distinguíveis de ruídos externos e de outros sons produzidos na região cervical, podendo, assim, ser utilizados em métodos de avaliação das disfagias.
\end{abstract}

Descritores: Deglutição; Auscultação/instrumentação; Diagnóstico por computador; Apneia; Fatores de tempo; Som/uso diagnóstico

\section{INTRODUÇÃO}

A ausculta cervical consiste em ouvir os sons da deglutição com auxílio de um instrumento de amplificação. Ela auxilia a avaliação da fase faríngea da deglutição por meio da tentativa da determinação da integridade do mecanismo de proteção das vias aéreas e estabelecimento dos tempos desses sons associados à deglutição ${ }^{(1)}$.

A deglutição pode ser didaticamente separada em fases de acordo com as regiões anatômicas envolvidas: fase oral, fase faríngea e fase esofágica. No início da fase faríngea da deglutição ocorre o fechamento do esfíncter velofaríngeo, das pregas vocais, vestibulares e ariepiglóticas, além da elevação e anteriorização da laringe e relaxamento e abertura do esfíncter esofágico superior. Entre esses mecanismos de proteção da via aérea contra a entrada de alimento está a apneia da deglutição,

Trabalho realizado no Departamento de Fonoaudiologia, Universidade Federal de São Paulo (SP), Brasil.

(1) Serviço Integrado de Fonoaudiologia do Hospital São Paulo, Universidade Federal de São Paulo - UNIFESP - São Paulo (SP), Brasil.

(2) Departamento de Fonoaudiologia, Universidade Federal de São Paulo UNIFESP - São Paulo (SP), Brasil.

(4) Curso de Especialização do Centro de Estudos da Voz São Paulo - CECEV - São Paulo (SP), Brasil.

Endereço para correspondência: Olívia Helena Gomes Patatas. R. Bom Jesus de Terra Preta, 62, Terra Preta, Mairiporã (SP), Brasil, CEP: 07600-000.

E-mail: oliviapatatas@ hotmail.com

Recebido em: 22/1/2010; Aceito em: 21/11/2010 durante a qual ciclos respiratórios são interrompidos após o fechamento das pregas vocais, imediatamente antes do início da fase faríngea. O sincronismo desses eventos é fundamental para o direcionamento do bolo para o esôfago e estômago, e prevenção da entrada do bolo na via aérea ${ }^{(2)}$.

Geralmente são encontrados dois, e algumas vezes três cliques distintos associados com a deglutição: dois cliques audíveis acompanhados de um sopro expiratório que se segue após a apneia da deglutição. No entanto, apesar das diversas tentativas de explicação da geração dos sons da deglutição já realizadas, nenhuma correlação com eventos fisiológicos pôde ser estabelecida com segurança $a^{(3-5)}$.

A maioria das deglutições desempenhadas por adultos saudáveis - 97\% - é seguida de expiração ${ }^{(6)}$, podendo, assim, ser considerada um bom marcador para o estudo da fase faríngea da deglutição. Sabe-se também que a retomada da respiração após a deglutição pode ocorrer com uma inspiração, em indivíduos normais ${ }^{(7)}$.

Conhecendo-se a proximidade entre a entrada do sistema digestório (esôfago) e a passagem para as vias aéreas inferiores (laringe), e a existência de um mesmo percurso através da faringe tanto para o ar quanto para o bolo deglutido, pode-se afirmar que uma deglutição segura requer precisão na coordenação entre deglutição e respiração. Falhas no funcionamento deste mecanismo de proteção ou alterações na sincronia adequada deste processo podem resultar em aspiração e complicações médicas secundárias ${ }^{(1)}$. Assim sendo, uma medição acurada do 
momento e da duração dos eventos associados à deglutição e à respiração pode ser um grande auxílio para a predição de uma deglutição segura.

Muitos profissionais da área acreditam que a ausculta cervical melhore a capacidade do profissional de predizer a aspiração e a penetração, a partir de inferências feitas com base nos sons percebidos durante a deglutição. Acredita-se que a ausculta cervical pode ser um método válido, e com boa capacidade de previsão, quando utilizada por um profissional capacitado. No entanto, a validade da ausculta cervical é ainda um tópico polêmico na literatura, uma vez que outros estudos demonstram pouca confiabilidade nas avaliações quanto à presença de aspiração feitas por meio deste método. Além disso, há muitas variáveis envolvidas na validade de sua realização, entre as quais estaria o instrumento que está sendo usado. $\mathrm{Na}$ maioria das vezes, este instrumento é um estetoscópio, o qual não permite uma análise mais aprofundada e acurada dos sons $\operatorname{captados}^{(8,9)}$.

O computador, capaz de digitalizar os sons e processar os ruídos produzidos pela deglutição em representações visuais, permite medidas exatas e, consequentemente, uma descrição mais precisa dos mesmos ${ }^{(1)}$, além de permitir a gravação e a revisão dos traçados acústicos quantas vezes forem necessárias. É possível que a análise feita com auxílio de um computador possa gerar aplicações clínicas de dados mais objetivos. Esses dados podem levar a comparações significativas da função da deglutição entre os sujeitos com deglutição normal e os sujeitos disfágicos, entre grupos de pacientes, e com o mesmo indivíduo em diferentes momentos ${ }^{(10)}$.

Como a morfofisiologia da deglutição se modifica com o crescimento e envelhecimento normais, e além da notória existência de diferenças anatômicas entre homens e mulheres, é necessário considerar a investigação de dados relativos à normalidade nas diferentes faixas etárias e entre os gêneros. O objetivo do presente estudo foi identificar a duração dos eventos acústicos captados durante a deglutição de indivíduos sem queixa, que sejam consistentes e comparáveis.

\section{MÉTODOS}

Esta pesquisa foi analisada e aprovada pelo Comitê de Ética em Pesquisa da UNIFESP, sob o protocolo CEP 1935/06. Todos os sujeitos e/ou seus responsáveis foram informados sobre os procedimentos e objetivos da pesquisa e assinaram o Termo de Consentimento Livre e Esclarecido.

A amostra deste estudo foi constituída por 164 indivíduos, sendo 88 mulheres e 76 homens, com idades entre 6 e 85 anos (média de 32,9 anos; mediana de 29 anos; moda de 10 anos): 61 indivíduos com idades entre 6 e 19 anos; 47 indivíduos, entre 20 e 39 anos; 22 com idades entre 40 e 59 anos; e 34 com 60 anos ou mais. Esses indivíduos foram recrutados em uma escola da rede pública de ensino, em uma organização voltada à terceira idade (Universidade Aberta à Terceira Idade) e em sala de espera de ambulatórios de um hospital público.

Os critérios de inclusão na amostra previam que os indivíduos não poderiam apresentar queixas atuais ou pregressas relativas à deglutição, risco ou histórico de disfagia, nem alterações morfológicas ou funcionais dos órgãos fonoarticulatórios.
Uma breve entrevista inicial foi realizada para investigação de possíveis impedimentos para inclusão na amostra. A entrevista foi realizada pessoalmente com os indivíduos adultos (Anexo 1) ou por meio de questionário respondido pelos pais ou responsáveis (Anexo 2), no caso de crianças. Durante a interação com o voluntário, a pesquisadora observou a presença de possíveis alterações nos órgãos envolvidos na fala e deglutição que impossibilitassem a inclusão do voluntário na amostra.

O sistema de captação de sons utilizado na pesquisa era formado por: um microfone de eletreto, do tipo gradiente de pressão, com resposta plana entre as frequências de $30 \mathrm{~Hz}$ a 12 $\mathrm{kHz}$; ligado a uma placa de som externa com plug compatível com entradas tipo USB (Universal Serial Bus). A faixa de frequência coberta pelo microfone visou incluir além dos sons da deglutição, os quais podem alcançar até $8 \mathrm{kHz}^{(11)}$, os sons da respiração, ainda mais agudos. Esse sistema de captação sonora foi conectado a entrada USB de um notebook (ECS G557). Os sons foram gravados a partir do software Sound Forge 4.5; a análise espectográfica, bem como acústica, foi realizada com auxílio, além desse mesmo software, do programa Praat 405 (ambos disponíveis para uso, sem custos, a partir da internet). Para a análise dos sons, utilizou-se o programa Praat 405 com as seguintes configurações: faixa de frequência de 0 a $5 \mathrm{kHz}$ e janela de $0,005 \mathrm{~s}$.

Os indivíduos foram posicionados sentados em cadeira, em frente a uma mesa, em ambiente silencioso. O microfone foi acoplado à pele do pescoço, usando fita adesiva e, sobre ela, uma faixa elástica ajustável com fechamento de velcro, no ponto localizado na borda lateral da traqueia imediatamente inferior à cartilagem cricoide, identificado por palpação da fonoaudióloga pesquisadora, por este ponto demonstrar a maior magnitude média e o menor desvio padrão da relação sinal/ ruído, como demonstrado anteriormente ${ }^{(3)}$. A faixa elástica foi ajustada ao pescoço de maneira confortável, com base no relato dos pacientes, garantindo-se que não houvesse pressão excessiva sobre a musculatura cervical.

Os indivíduos foram orientados a evitar falar, pigarrear ou produzir ruídos que pudessem interferir nas gravações. Além disso, deveriam aguardar o sinal da pesquisadora para dar início a cada uma das sequências de deglutição descritas acima.

Para a deglutição "a seco", os indivíduos foram orientados a “juntar saliva na boca” e então engolir, repetindo, em seguida, o procedimento outras duas vezes. No caso das deglutições com $5 \mathrm{ml}$ ou $10 \mathrm{ml}$ de água, três copos descartáveis pequenos contendo os volumes de água previamente medidos em seringa graduada estavam sobre a mesa ao alcance do indivíduo. Ao sinal da pesquisadora, o indivíduo deveria pegar o primeiro copo, levá-lo à boca e deglutir todo o volume de água em uma só deglutição, evitando deixar resíduo do líquido no copo; seguindo, então para os próximos copos. Nenhuma interferência da pesquisadora quanto à postura e/ou movimentação, inclusive de cabeça, foi realizada durante as gravações, buscando manter o padrão habitual do paciente durante as deglutições.

Cada indivíduo realizou nove deglutições, sendo: três deglutições "a seco"; três deglutições de $5 \mathrm{ml}$ de água; e três deglutições de $10 \mathrm{ml}$ água.

A ordem de apresentação das ofertas foi randomizada como se segue, para cada faixa etária com extensão de dez 
anos (0-10 anos, 11-20 anos, e assim sucessivamente), visando detectar possíveis variações nos resultados devidas à ordem de apresentação:

- Sequência 1: três deglutições de saliva; três de $5 \mathrm{ml}$ de água; três de $10 \mathrm{ml}$ de água;

- Sequência 2: três deglutições de saliva; três de $10 \mathrm{ml}$ de água; três de $5 \mathrm{ml}$ de água;

- Sequência 3: três deglutições de $5 \mathrm{ml}$ de água; três de 10 ml de água; três de saliva;

- Sequência 4: três deglutições de $5 \mathrm{ml}$ de água; três de saliva; três de $10 \mathrm{ml}$ de água;

- Sequência 5: três deglutições de $10 \mathrm{ml}$ de água; três de 5 ml de água; três de saliva;

- Sequência 6: três deglutições de $10 \mathrm{ml}$ de água; três de saliva; três de $5 \mathrm{ml}$ de água.

A randomização foi realizada de acordo com a ordem de chegada dos voluntários na sala de gravação; assim, o primeiro foi submetido à sequência 1; o segundo, à sequência 2; até que no sexto voluntário, retornou-se a sequência 1; e assim, sucessivamente.

Os sons das deglutições gravados foram analisados com base em procedimento já descrito na literatura ${ }^{(1)}$, porém, modificado (Figura 1).

Todos os ruídos que não correspondessem ao padrão dos sons da deglutição, já descrito na literatura ${ }^{(1,4-6,12,13)}$ e observado ao longo das análises deste trabalho, foram desprezados du- rante a análise. Dois indivíduos foram excluídos da amostra, pois nenhuma das deglutições gravadas foi passível de análise, devido a ruídos excessivos, de origem desconhecida, que interferiram nas gravações. Das 1476 deglutições restantes, 46 deixaram de ser analisadas por motivos como: deglutições não apresentavam traçado compatível com o descrito na literatura; não detecção do ruído respiratório; ruídos, interferências e artefatos; fracionamento do bolo durante a deglutição.

O julgamento dos componentes significativos do som registrado foi feito com base nas análises visual e auditiva simultâneas do sinal. A inspeção dos sinais gravados foi repetida diversas vezes, enquanto se buscava identificar o momento de cessação da respiração, no qual a intensidade do som respiratório deixa de ser auditivamente percebido e o padrão espectrográfico revela significativa mudança visual; além de se realizar a marcação das explosões da deglutição, percebidas devido à mudança abrupta do sinal basal, com incremento significativo da intensidade do som e mudança no gráfico de intensidade da espectrografia, no qual se observa a formação de dois picos de intensidade. Quando necessário, para melhor análise auditiva, a gravação sofreu aumento no volume geral (ganho) durante as análises, sem distorções de qualquer componente ou parte do som isoladamente.

Para a análise estatística, foi definido um nível de significância, ou seja, o erro estatístico tolerável nas análises, de 0,05 (5\%). Todos os intervalos de confiança foram construídos com $95 \%$ de confiança estatística. Foram utilizados testes e técnicas estatísticas não-paramétricas visto que as condições para a utilização de téc-

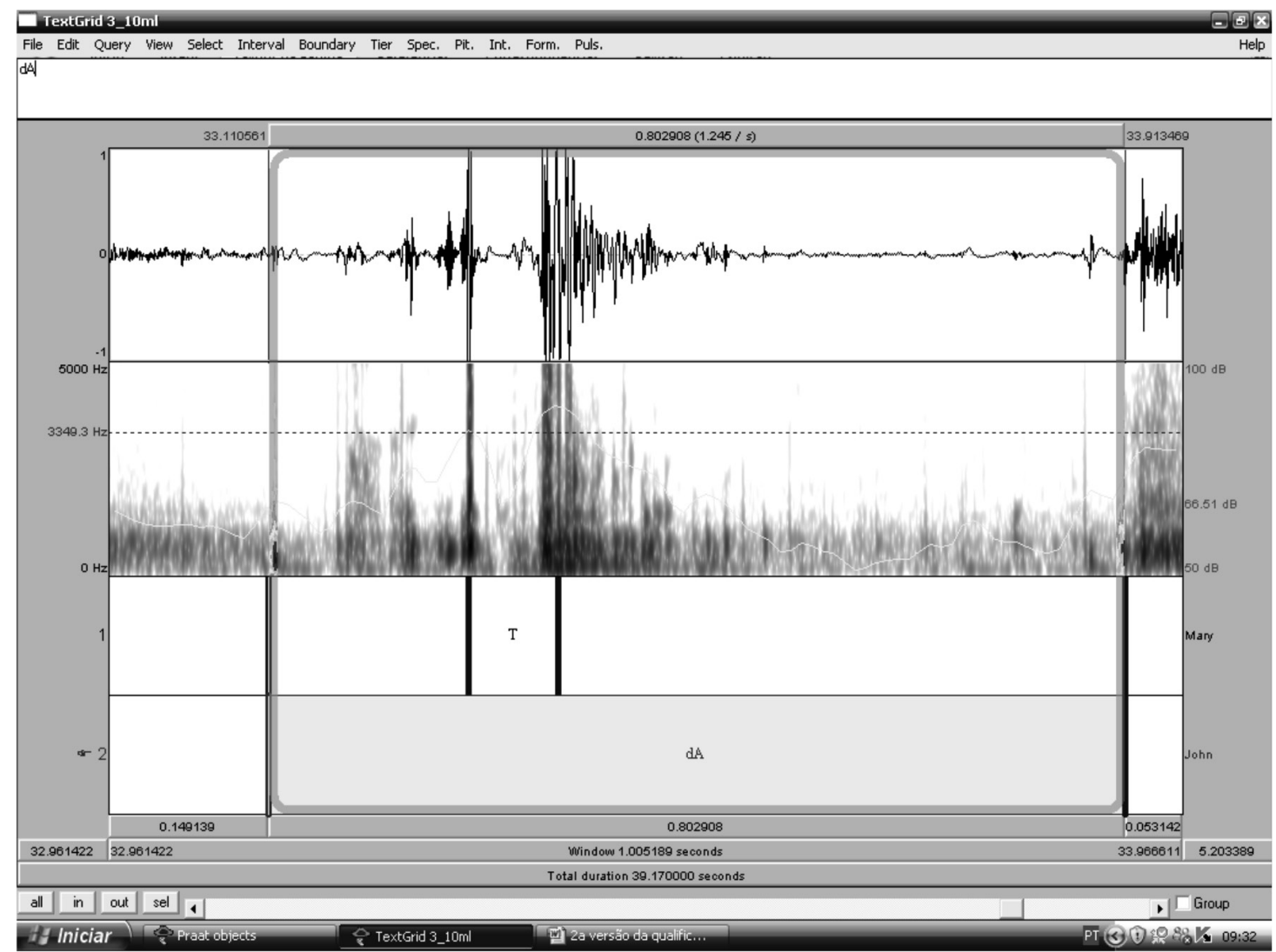

Legenda: $\mathrm{T}$ = tempo entre as duas explosões da deglutição; $\mathrm{dA}=$ apneia da deglutição

Figura 1. Exemplo da análise do traçado espectrográfico dos sons de uma deglutição 
Tabela 1. Análise descritiva dos aspectos temporais relacionados aos sons da deglutição de saliva

\begin{tabular}{|c|c|c|c|c|c|c|c|c|c|}
\hline \multirow{2}{*}{ Descritiva } & \multicolumn{3}{|c|}{ Saliva } & \multicolumn{3}{|c|}{$5 \mathrm{ml}$} & \multicolumn{3}{|c|}{$10 \mathrm{ml}$} \\
\hline & $\mathrm{T}(\mathrm{ms})$ & $\mathrm{dA}(\mathrm{ms})$ & $\mathrm{T} / \mathrm{dA}(\mathrm{ms})$ & $\mathrm{T}(\mathrm{ms})$ & $\mathrm{dA}(\mathrm{ms})$ & $\mathrm{T} / \mathrm{dA}(\mathrm{ms})$ & $\mathrm{T}(\mathrm{ms})$ & $\mathrm{dA}(\mathrm{ms})$ & $\mathrm{T} / \mathrm{dA}(\mathrm{ms})$ \\
\hline Média & 201,3 & 1774 & 0,152 & 179,6 & 1436 & 0,177 & 176,5 & 1531 & 0,158 \\
\hline Mediana & 188,1 & 1638 & 0,136 & 174,7 & 1132 & 0,150 & 163,3 & 1363 & 0,141 \\
\hline DP & 82,7 & 825 & 0,080 & 76,4 & 906 & 0,119 & 83,3 & 809 & 0,103 \\
\hline CV & $41,1 \%$ & $46,5 \%$ & $52,9 \%$ & $42,6 \%$ & $63,0 \%$ & $67,0 \%$ & $47,2 \%$ & $52,8 \%$ & $65,1 \%$ \\
\hline Q1 & 146,8 & 1170 & 0,096 & 135,9 & 892 & 0,106 & 123,8 & 948 & 0,091 \\
\hline Q3 & 227,5 & 2204 & 0,185 & 199,3 & 1791 & 0,213 & 200,5 & 1882 & 0,189 \\
\hline Min & 75,6 & 472 & 0,026 & 52,1 & 305 & 0,039 & 36,7 & 478 & 0,015 \\
\hline Max & 486,3 & 5380 & 0,596 & 571,6 & 6808 & 1,111 & 631,1 & 5512 & 0,791 \\
\hline $\mathrm{N}$ & 159 & 159 & 159 & 159 & 160 & 159 & 159 & 159 & 159 \\
\hline IC & 12,8 & 128,2 & 0,012 & 11,9 & 140,3 & 0,018 & 12,9 & 125,8 & 0,016 \\
\hline
\end{tabular}

Legenda: DP = desvio-padrão; CV = coeficiente de variação; Q1 = primeiro quartil; Q3 = terceiro quartil; Min = mínimo; Max = máximo; IC = intervalo de confiança

nicas e testes paramétricos, como a normalidade da distribuição e a homocedasticidade (homogeneidade das variâncias), não foram encontradas no conjunto de dados. Além da análise descritiva dos dados, foram utilizados os seguintes testes: Kruskal-Wallis (para comparar as sequências de oferta), Correlação de Spearman (para medir o grau de relação entre a idade e os tempos mensurados), Mann-Whitney (para comparar os resultados entre os sexos), o teste de Friedman e o teste de Wilcoxon (ambos para comparar os resultados entre saliva, $5 \mathrm{ml} \mathrm{e} 10 \mathrm{ml}$ ).

\section{RESULTADOS}

A partir do teste de Kruskal-Wallis para comparação das sequências randomizadas de obtenção das deglutições, observou-se que não houve diferença entre as sequências de obtenção das deglutições e as medidas temporais realizadas, a saber: tempo decorrido entre os picos das duas explosões da deglutição (T), duração da apneia da deglutição (dA) e índice obtido pela relação T/dA.

Na Tabela 1 e nas Figuras 2 e 3 estão representadas as análises dos aspectos temporais relacionados aos sons da deglutição.

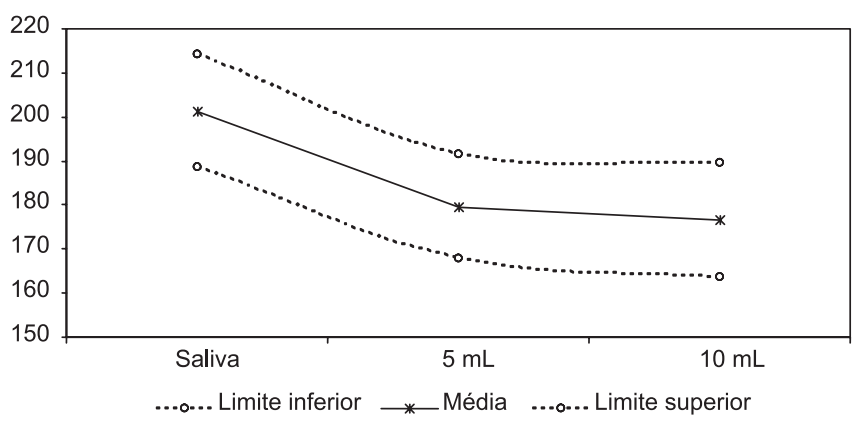

Figura 2. Intervalo de confiança para T, considerando os aspectos temporais relacionados aos sons da deglutição

Os valores de p das Tabelas 2 e 3 revelam que houve diferença entre os volumes deglutidos com relação às medidas temporais realizadas, a saber, $\mathrm{T}$ e dA isoladamente.

Quando se trata do intervalo entre as explosões da deglutição $(\mathrm{T})$, verificou-se que quanto maior o volume do bolo deglutido, menor o tempo decorrido entre as explosões. Já o

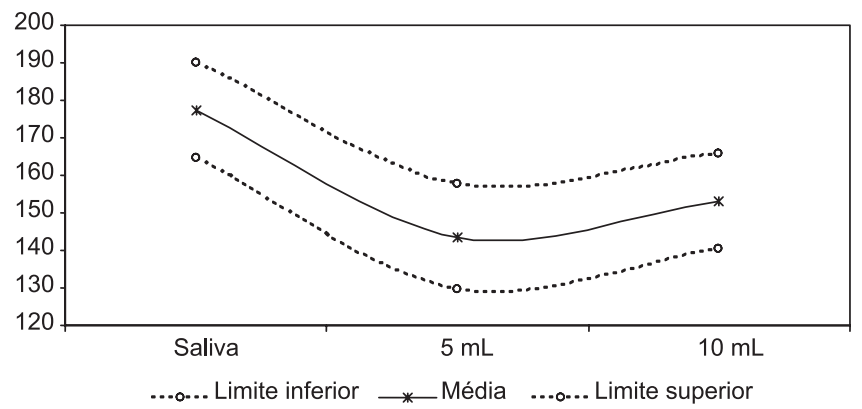

Figura 3. Intervalo de confiança para dA, considerando os aspectos temporais relacionados aos sons da deglutição

resultado da análise da duração da apneia da deglutição vemos que a menor duração foi na oferta de $5 \mathrm{ml}$ de água, seguida por $10 \mathrm{ml}$ e, por último, saliva.

A Tabela 3 indica o grau de relação entre a idade dos indivíduos e os tempos mensurados.

As correlações entre idade e tempos medidos foram consideradas péssimas ou ruins, ou seja, a idade dos indivíduos não influenciou os tempos descritos entre as duas explosões da deglutição ou a duração da apneia da deglutição.

A comparação dos resultados entre os gêneros está representada nas Figuras 4 e 5, e na Tabela 4.

Somente quanto ao índice T/dA nas deglutições de $5 \mathrm{ml}$ encontrou-se diferença entre os gêneros, isto é, os homens apresentaram índices maiores do que as mulheres.

\section{DISCUSSÃO}

Os tempos médios decorridos entre as explosões da deglutição $(\mathrm{T})$, medidos desde o pico da primeira explosão ao pico da segunda, foram maiores quanto maiores eram os volumes deglutidos. Medidas específicas da duração das deglutições de 260 indivíduos não-disfágicos com metodologia semelhante àquela utilizada no presente estudo foram realizadas por outro autor que encontrou intervalos entre as explosões (definido como T) próximos aos encontradas neste estudo ${ }^{(1)}$.

Três trabalhos referem durações relativas ao tempo decorrido entre as duas explosões da deglutição menores que as descritas aqui( ${ }^{(3,13,14)}$. Essa diferença pode ser justificada pelas 
Tabela 2. Comparação entre os volumes deglutidos

\begin{tabular}{|c|c|c|c|c|c|c|c|c|c|}
\hline Doses & & Média & Mediana & DP & Q1 & Q3 & $\mathrm{n}$ & IC & Valor de $p$ \\
\hline & Saliva & 201,3 & 188,1 & 82,7 & 146,8 & 227,5 & 159 & 12,8 & \\
\hline \multirow[t]{3}{*}{$\mathrm{T}(\mathrm{ms})$} & $5 \mathrm{ml}$ & 179,6 & 174,7 & 76,4 & 135,9 & 199,3 & 159 & 11,9 & $0,007^{*}$ \\
\hline & $10 \mathrm{ml}$ & 176,5 & 163,3 & 83,3 & 123,8 & 200,5 & 159 & 12,9 & \\
\hline & Saliva & 1774 & 1638 & 825 & 1170 & 2204 & 159 & 128,2 & \\
\hline \multirow[t]{3}{*}{$\mathrm{dA}(\mathrm{ms})$} & $5 \mathrm{ml}$ & 1436 & 1132 & 906 & 892 & 1791 & 160 & 140,3 & $<0,001^{*}$ \\
\hline & $10 \mathrm{ml}$ & 1531 & 1363 & 809 & 948 & 1882 & 159 & 125,8 & \\
\hline & Saliva & 0,152 & 0,136 & 0,080 & 0,096 & 0,185 & 159 & 0,012 & \\
\hline \multirow[t]{2}{*}{$\mathrm{T} / \mathrm{dA}(\mathrm{ms})$} & $5 \mathrm{ml}$ & 0,177 & 0,150 & 0,119 & 0,106 & 0,213 & 159 & 0,018 & $0,017^{\star}$ \\
\hline & $10 \mathrm{ml}$ & 0,158 & 0,141 & 0,103 & 0,091 & 0,189 & 159 & 0,016 & \\
\hline
\end{tabular}

*Valores significativos $(p \leq 0,05)-$ Teste de Friedman

Legenda: $Q 1$ = primeiro quartil; Q3 = terceiro quartil; IC = intervalo de confiança; $T$ = tempo decorrido entre os picos das duas explosões da deglutição; dA = duração da apneia da deglutição; $\mathrm{T} / \mathrm{dA}$ = relação entre tempo decorrido entre as explosões da deglutição e a apneia da deglutição; DP = desvio-padrão

Tabela 3. Correlação idade $x$ tempo nas deglutições de saliva

\begin{tabular}{llcc}
\hline \multirow{2}{*}{ Tempo } & & \multicolumn{2}{c}{ Idade } \\
\cline { 3 - 4 } & & Correlação & Valor de $\mathrm{p}$ \\
\hline \multirow{2}{*}{ Saliva } & $\mathrm{T}(\mathrm{ms})$ & $6,3 \%$ & 0,431 \\
& $\mathrm{dA}(\mathrm{ms})$ & $23,6 \%$ & $0,003^{*}$ \\
& $\mathrm{~T} / \mathrm{dA}(\mathrm{ms})$ & $-10,7 \%$ & 0,180 \\
\hline \multirow{3}{*}{$5 \mathrm{ml}$} & $\mathrm{T}(\mathrm{ms})$ & $15,1 \%$ & 0,057 \\
& $\mathrm{dA}(\mathrm{ms})$ & $16,1 \%$ & $0,041^{*}$ \\
& $\mathrm{~T} / \mathrm{dA}(\mathrm{ms})$ & $0,6 \%$ & 0,936 \\
\hline \multirow{2}{*}{$10 \mathrm{ml}$} & $\mathrm{T}(\mathrm{ms})$ & $20,7 \%$ & $0,009^{*}$ \\
& $\mathrm{dA}(\mathrm{ms})$ & $12,3 \%$ & 0,123 \\
& $\mathrm{~T} / \mathrm{dA}(\mathrm{ms})$ & $6,1 \%$ & 0,446 \\
\hline
\end{tabular}

* Valores significativos $(p \leq 0,05)$ - Teste de Correlação de Spearman

Legenda: $T$ = tempo decorrido entre os picos das duas explosões da deglutição; $\mathrm{dA}=$ duração da apneia da deglutição; $\mathrm{T} / \mathrm{dA}=$ relação entre tempo decorrido entre as explosões da deglutição e a apneia da deglutição

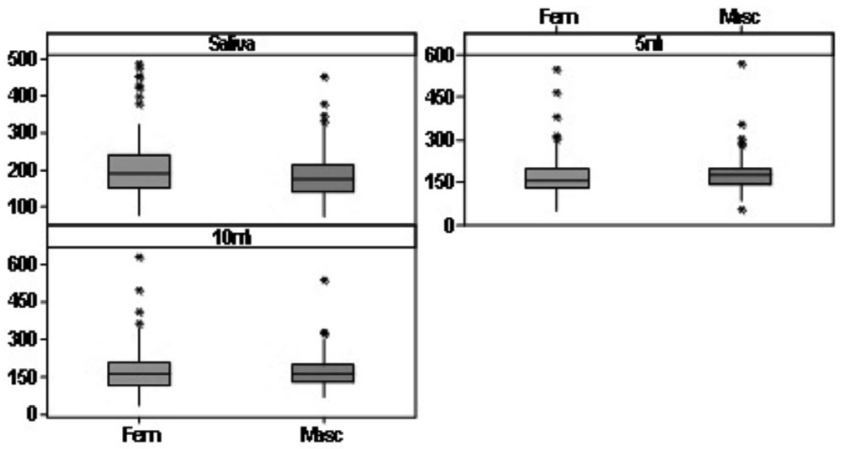

Legenda: Fem = gênero feminino; Masc = gênero masculino

Figura 4. Comparação entre gêneros em relação ao tempo decorrido entre os picos das duas explosões da deglutição (ms)

diferenças entre as metodologias empregadas, uma vez que aqueles estudos mensuraram apenas os intervalos entre as duas explosões, ou seja, os tempos descritos pelas explosões propriamente ditas foram excluídos do intervalo considerado, enquanto neste trabalho os picos das explosões foram utilizados como marcadores, sendo incluídas partes significativas delas na duração encontrada.

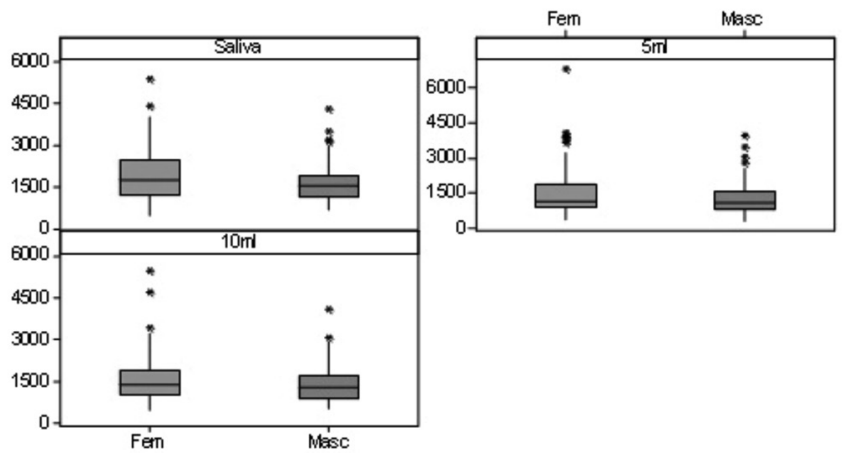

Legenda: Fem = gênero feminino; Masc = gênero masculino Figura 5. Comparação entre gêneros em relação à duração da apneia da deglutição (ms)

Tabela 4. Comparação da relação $\mathrm{T} / \mathrm{dA}$, considerando o gênero dos indivíduos

\begin{tabular}{lcccccc}
\hline \multirow{2}{*}{ T/dA (ms) } & \multicolumn{2}{c}{ Saliva } & \multicolumn{2}{c}{$5 \mathrm{ml}$} & \multicolumn{2}{c}{$10 \mathrm{ml}$} \\
& Fem & Masc & Fem & Masc & Fem & Masc \\
\hline Média & 0,154 & 0,149 & 0,159 & 0,198 & 0,150 & 0,166 \\
Mediana & 0,137 & 0,135 & 0,134 & 0,170 & 0,134 & 0,142 \\
DP & 0,088 & 0,071 & 0,091 & 0,143 & 0,094 & 0,111 \\
Q1 & 0,094 & 0,100 & 0,099 & 0,120 & 0,079 & 0,104 \\
Q3 & 0,187 & 0,179 & 0,191 & 0,222 & 0,187 & 0,187 \\
$\mathrm{n}$ & 86 & 73 & 87 & 72 & 86 & 73 \\
IC & 0,019 & 0,016 & 0,019 & 0,033 & 0,020 & 0,026 \\
Valor de p & \multicolumn{2}{c}{0,953} & $0,014^{*}$ & 0,287 \\
\hline
\end{tabular}

Legenda: DP = desvio-padrão; $\mathrm{Q} 1$ = primeiro quartil; $\mathrm{Q} 3$ = terceiro quartil; $\mathrm{IC}=$ intervalo de confiança; $\mathrm{T}=$ tempo decorrido entre os picos das duas explosões da deglutição; $\mathrm{dA}=$ duração da apneia da deglutição; Fem = gênero feminino; Masc = gênero masculino; $\mathrm{T} / \mathrm{dA}$ = relação entre tempo decorrido entre as explosões da deglutição e a apneia da deglutição

Por outro lado, diversos estudos mostram durações dos sons da deglutição significativamente maiores que os tempos encontrados aqui, variando entre 250 e $800 \mathrm{~ms}^{(3,15-18)}$. Há diversas metodologias e materiais com tecnologias bastante distintas que podem ter propiciado diferentes graus de precisão na medição dos tempos. Encontram-se na literatura captações dos sons realizadas com microfones adaptados em bifurcações de estetos- 
cópios, microfones de contato, acelerômetros e Sonar Doppler, e registradas em equipamentos de tecnologia obsoleta como gravadores de fita cassete e osciloscópios, indo até sofisticadas análises computadorizadas. Além disso, não há consenso entre os pesquisadores do que pode ser considerado início e fim da deglutição, faltando o estabelecimento de marcos claros e reprodutíveis para a realização das medidas de tempo.

Quanto à duração da apneia da deglutição (dA), outros autores também realizaram mensurações utilizando Sonar Doppler. Esses autores mediram desde o início da apneia até a retomada da respiração e encontraram durações semelhantes às deste trabalho ${ }^{(19)}$.

A duração maior da apneia nas deglutições de saliva encontrada no presente estudo pode se dever ao fato de que essas deglutições originaram, no geral, registros de baixa intensidade, inclusive para os sons da respiração, que, quanto mais próximos do início da deglutição perdem intensidade significativamente, dificultando, em alguns casos, o estabelecimento exato da interrupção da respiração. Estudos que realizaram a análise do fluxo aéreo durante a deglutição, mais precisas do que as obtidas no presente estudo, revelaram durações menores, provavelmente representando melhor a realidade existente na população ${ }^{(6,20,21)}$.

Outro estudo, no qual videodeglutogramas associados à monitoração do fluxo aéreo nasal foram realizados, encontrou apneias da deglutição maiores que aqueles mensuradas no presente trabalho, com duração média de 2 segundos ${ }^{(7)}$.

As gravações e a análise dos sons realizadas neste estudo talvez não sejam ainda o método mais apurado para a análise da apneia da deglutição, uma vez que não se capta diretamente a cessação do fluxo aéreo, mas sim a redução do ruído respiratório; na maior parte dos casos, o microfone de contato gera um sinal de baixa intensidade para a respiração.

Para os valores dos índices obtidos a partir da relação entre T/dA, para as deglutições de saliva, de $5 \mathrm{ml}$ de água e de $10 \mathrm{ml}$ de água, não se encontram correspondentes na literatura, não sendo possível a comparação com outros estudos. Conforme a hipótese já levantada ${ }^{(1)}$, quanto mais próximo de um esses valores se encontrarem, maiores seriam as chances de os indivíduos apresentarem broncoaspiração, uma vez que estariam iniciando a apneia quando a deglutição, mais especificamente a fase faríngea, já estaria em processo, ou retomando a respiração muito próximo ao momento final do trânsito faríngeo do bolo. Pode-se notar que as deglutições de indivíduos não disfágicos deste estudo, apesar da variação intra e inter sujeitos, apresentam-se extremamente seguras, uma vez que os valores encontrados estão muito abaixo de um. Levando-se em consideração as médias calculadas e o intervalo de confiança para essa relação, sugere-se que indivíduos que apresentam valores fora dessa faixa devem ser avaliados com cautela, devido ao risco potencial para broncoaspiração.

Nossos achados corroboram outro estudo ${ }^{(10)}$, no qual os autores afirmam que o tempo de trânsito faríngeo é razoavelmente constante nos sujeitos normais e não mudou significativamente com a mudança do tamanho do bolo. No entanto, os autores perceberam mudanças nos tempos de cada gesto da deglutição com a mudança no tamanho do bolo, os quais provavelmente representem acomodações feitas para lidar com volumes maiores de bolo, visando manter uma duração constante do trânsito faríngeo. Dois trabalhos notaram que a variação no volume do bolo afetou significativamente as medidas de tempo realizadas ${ }^{(2,23)}$. Vale ressaltar que esses estudos utilizaram bolos com volumes que diferiam mais entre si do que o presente estudo que variou apenas em $5 \mathrm{ml}$ o volume entre os bolos oferecidos, podendo essa diferença não ser suficiente para uma variação significativa nas medidas do ponto de vista estatístico. Um exemplo é o trabalho que revelou que a média das apneias da deglutição para $5 \mathrm{ml}$ de água espessada foi significativamente menor que para $15 \mathrm{ml}$ e $20 \mathrm{ml}$, mas não o foi quando comparada a $10 \mathrm{ml}^{(24)}$.

Resultados opostos foram obtidos por outros autores ao relatarem que a duração do som da deglutição, de maneira geral, diminuiu com volumes maiores de bolo, resultado esse que difere daquele encontrado no presente estudo ${ }^{(18)}$. Não se pode perder de vista que, em cada pesquisa citada, os marcos de referência considerados para a medição das durações dos sons da deglutição diferiram, o que invalida a comparação direta entre os resultados encontrados.

Quanto à duração da apneia, nossos achados corroboram os dados da literatura ao revelarem que houve diferença na duração da apneia da deglutição entre as deglutições dos diferentes volumes, principalmente quando se compara saliva com as deglutições de água $(5 \mathrm{ml} \mathrm{e} 10 \mathrm{ml})$. Outro estudo avaliou quarenta adultos normais com bolos de diferentes consistências e volumes $(5 \mathrm{ml}$ e $20 \mathrm{ml})$. Os bolos maiores eliciaram a apneia da deglutição mais cedo que os menores e a duração da apneia apenas aumentou com o incremento de $15 \mathrm{ml}$ no volume do bolo ${ }^{(25)}$. De modo semelhante, já foi relatado que a duração da apneia somente apresentou aumento com o incremento no volume do bolo quando esse passou de $5 \mathrm{ml}$ para 15 ou $20 \mathrm{ml}$. A média das apneias para bolos de $10 \mathrm{ml}$ não diferiram das de $5 \mathrm{ml}^{(24)}$.

O presente estudo revelou diferenças quanto aos tempos absolutos (T e dA) entre as deglutições de saliva e as deglutições de água, sendo a duração do intervalo entre as explosões da deglutição (T) e a duração da apneia da deglutição (dA) discretamente maiores para as deglutições de saliva; entretanto, não houve diferença estatística ao relacionar-se T/dA, obtendo-se um índice. Outros autores relataram que as deglutições "a seco" tenderam a ser maiores em duração do que as deglutições de água. Além disso, elas não exibiram o padrão de características esperado, isto é, duas explosões principais ${ }^{(12)}$.

Não se pôde afirmar que há correlação entre a faixa etária e os tempos mensurados a partir dos sons captados durante a deglutição. No entanto, diversos estudos sugerem que o envelhecimento acarreta a redução da velocidade dos mecanismos da deglutição, o que poderia levar a modificações nos aspectos temporais dos sons da deglutiç̧ão ${ }^{(26-30)}$. Autores relataram que a duração dos sons da deglutição de $5 \mathrm{ml}$ foi significativamente menor para os sujeitos entre 18 e 35 anos de idade do que para aqueles com 60 anos ou mais ${ }^{(18)}$. Outro trabalho relatou um aumento na duração e diminuição na intensidade do sinal com o aumento da idade ${ }^{(16)}$.

Em contraponto, outros autores, em estudo videofluoroscópico, notaram que o tempo de trânsito faríngeo não aumentou com $\mathrm{o}$ avanço da idade. Os autores acreditam que o tempo prolongado de trânsito faríngeo observado em outros estudos, pode estar mais relacionado a comorbidades do que à idade dos sujeitos ${ }^{(6)}$. 
Os estudos referidos realizaram medidas relativas à duração total do sinal da deglutição, enquanto o presente estudo mediu a distância, em termos de tempo, apenas entre os picos das duas principais explosões da deglutição. Talvez, a redução da velocidade na movimentação das estruturas observada pelos autores gere um aumento do tempo total da deglutição pelo aumento do tempo necessário ao alcance do pico da primeira explosão, desde o início da perturbação do sinal basal prédeglutição; mas, a partir daí, não se observe interferência no intervalo entre a primeira e a segunda explosão. Tal raciocínio encontra-se em concordância com a hipótese levantada por outra pesquisa, na qual os autores relatam que as mudanças neuromotoras cogitadas como contribuintes para um aumento na duração da fase faríngea da deglutição acarretam: tempo de resposta aumentado para o disparo do reflexo da deglutição, tempo aumentado de movimentação da laringe até sua posição superior/anterior devido à posição mais baixa da laringe no pescoço; velocidade e amplitude peristálticas reduzidas possivelmente relacionadas à incidência aumentada de artrite das vértebras cervicais ${ }^{(16)}$. A hipótese levantada é de que essa redução da velocidade dos eventos da deglutição, associada à idade, poderia ser também responsável pelo tempo aumentado entre o início do sinal da deglutição e o ponto no qual o pico de intensidade do sinal ocorre.

Com relação aos gêneros, somente quanto ao índice T/dA, nas deglutições de $5 \mathrm{ml}$, encontrou-se diferença estatisticamente significativa, isto é, os homens apresentaram índices maiores do que as mulheres: 0,198 e 0,159, respectivamente (Tabela 5). Não se encontrou nenhum dado na literatura consultada que justificasse tal achado ou que permitisse a elaboração de uma hipótese plausível para essa diferença. Quanto às medidas absolutas, intervalo entre os picos das duas explosões da deglutição (T) e duração da apneia da deglutição, não houve diferença estatística entre homens e mulheres. Esses achados corroboram os resultados de outros encontrados na literatura recente ${ }^{(13,16,19)}$.

Por outro lado, já foi relatado que a duração dos sons da deglutição de mulheres foi menor que a de homens. Deve-se considerar que o tamanho da amostra utilizada naqueles estudos pode não propiciar dados representativos da realidade ${ }^{(17)}$.

De maneira oposta, outro estudo sugere que os sons da deglutição nas mulheres, bem como a apneia da deglutição, são mais longos que nos homens ${ }^{(18,24,25)}$.

Os resultados encontrados neste estudo não podem ser tomados irrestritamente como padrão de normalidade, visto que o tamanho da amostra não caracteriza uma normatização. Apesar disso, o estudo aponta para a possível aplicabilidade clínica dos parâmetros temporais relativos aos sons da deglutição, analisados por meio de ausculta cervical computadorizada. Apesar das variações inter e intra-sujeitos, os sons da deglutição apresentam um padrão característico e reprodutível, sendo distinguíveis de ruídos externos e de outros sons produzidos na região cervical, podendo contribuir com dados objetivos à avaliação clínica fonoaudiológica da deglutição, além de haver a possibilidade de sua utilização como biofeedback na terapia fonoaudiológica.

Novos estudos, com amostra ampliada, visando a normatização desses parâmetros, bem como sua comparação com medidas realizadas entre indivíduos disfágicos, considerandose as diferentes etiologias, possibilitariam a constatação da abrangência e real aplicação.

\section{CONCLUSÃO}

Com base no objetivo de investigar parâmetros relacionados à duração dos eventos acústicos captados durante a deglutição de indivíduos sem queixa, que sejam consistentes e comparáveis, concluímos que as durações médias dos tempos decorridos entre os picos das duas explosões da deglutição (T) tenderam a ser menores quanto maior o volume deglutido. As durações médias das apneias da deglutição (dA) foram cada vez maiores quanto maior o volume deglutido. Por fim, o índice T/dA médio foi maior para o volume de $5 \mathrm{ml}$, seguido pela deglutição de $10 \mathrm{ml}$ e, por último, o menor valor foi na deglutição de saliva.

A idade não influenciou os tempos mensurados. Já com relação ao gênero, somente quanto ao índice T/dA nas deglutições de $5 \mathrm{ml}$ há de se levar em consideração o gênero do indivíduo, visto que os homens apresentaram índices maiores do que as mulheres. Quanto às medidas absolutas, T e dA, não houve diferença entre os gêneros.

\section{AGRADECIMENTOS}

Ao Conselho Nacional de Desenvolvimento Científico e Tecnológico - CNPq, pelo apoio concedido por meio do processo 133896/2007-2. 


\begin{abstract}
Purpose: To investigate the consistency of duration and reproducibility of the pattern of acoustical events related to the swallowing of individuals without complaints. Methods: Participants were 88 women and 76 men with ages between 6 and 85 years. With a microphone attached to the neck and connected to a computer, each of them performed nine swallowings of saliva, of $5 \mathrm{ml}$ of water, and of $10 \mathrm{ml}$ of water. Based on the acoustic registration of the sounds recorded, the duration of the interval between the peaks of the two main swallowing clicks (T), of the swallowing apnea (dA), and of the relationship between them (T/dA). Results: No differences were found regarding the mean duration between the peaks of the two swallowing clicks ( $\mathrm{T}$ ), the mean duration of the swallowing apnea (dA), and the relationship T/dA in the different age ranges and between genders, except for the T/dA index for the swallowing of $5 \mathrm{ml}$ of water. There was no correlation between age and the measured parameters. Conclusion: The swallowing sounds present a characteristic reproducible pattern, which is distinct both from external noises and from other sounds produced in the cervical region. Therefore, they may be used in dysphagia evaluation methods.
\end{abstract}

Keywords: Deglutition; Auscultation/instrumentation; Diagnosis, computer-assisted; Apnea; Time factors; Sounds/diagnostic use

\title{
REFERÊNCIAS
}

1. Furkim AN, Santini CS. Disfagias orofaríngeas. Carapicuíba: Pró-Fono; 1999.

2. McConnel FM, Cerenko D, Jackson RT, Guffin TN Jr. Timing of major events of pharyngeal swallowing. Arch Otolaryngol Head Neck Surg. 1988;114(12):1413-8.

3. Takahashi K, Groher ME, Michi K. Methodology for detecting swallowing sounds. Dysphagia. 1994;9(1):54-62.

4. Hamlet SL, Nelson RJ, Patterson RL. Interpreting the sounds of swallowing: fluid flow through the cricopharyngeus. Ann Otol Rhinol Laryngol. 1990;99(9 Pt 1):749-52.

5. Selley WG, Ellis RE, Flack FC, Bayliss CR, Pearce VR. The synchronization of respiration and swallow sounds with videofluoroscopy during swallowing. Dysphagia. 1994;9(3):162-7.

6. Perlman AL, Ettema SL, Barkmeier J. Respiratory and acoustic signals associated with bolus passage during swallowing. Dysphagia. 2000;15(2):89-94.

7. Martin-Harris B, Brodsky MB, Price CC, Michel Y, Walters B. Temporal coordination of pharyngeal and laryngeal dynamics with breathing during swallowing: single liquid swallows. J Appl Physiol. 2003;94(5):1735-43.

8. Zenner PM, Losinski DS, Mills RH. Using cervical auscultation in the clinical dysphagia examination in long-term care. Dysphagia. 1995;10(1):27-31.

9. Stroud AE, Lawrie BW, Wiles CM. Inter- and intra-rater reliability of cervical auscultation to detect aspiration in patients with dysphagia. Clin Rehabil. 2002;16(6):640-5.

10. Kendall KA, McKenzie S, Leonard RJ, Gonçalves MI, Walker A. Timing of events in normal swallowing: a videofluoroscopic study. Dysphagia. 2000;15(2):74-83.

11. Cichero JA, Murdoch BE. The physiologic cause of swallowing sounds: answers from heart sounds and vocal tract acoustics. Dysphagia. 1998;13(1):39-52.

12. Mackowiak RC, Brenman HS, Friedman MH. Acoustic profile of deglutition. Proc Soc Exp Biol Med. 1967;125(4):1149-52.

13. Morinière $S$, Beutter $P$, Boiron $M$. Sound component duration of healthy human pharyngoesophageal swallowing: a gender comparison study. Dysphagia. 2006;21(3):175-82.

14. Morinière S, Boiron M, Alison D, Makris P, Beutter P. Origin of the sound components during pharyngeal swallowing in normal subjects. Dysphagia. 2008;23(3):267-73.

15. Logan WJ, Kavanagh JF, Wornall AW. Sonic correlates of human deglutition. J Appl Physiol. 1967;23(2):279-84.
16. Youmans SR, Stierwalt JA. An acoustic profile of normal swallowing. Dysphagia. 2005;20(3):195-209.

17. Takahashi K, Groher ME, Michi K. Symmetry and reproducibility of swallowing sounds. Dysphagia. 1994;9(3):168-73.

18. Cichero JA, Murdoch BE. Acoustic signature of the normal swallow: characterization by age, gender, and bolus volume. Ann Otol Rhinol Laryngol. 2002;111(7 Pt 1):623-32.

19. Santos RS, Macedo Filho ED. Sonar doppler como instrumento de avaliação da deglutição. Arq Int Otorrinolaringol. 2006;10(3):182-91.

20. Selley WG, Flack FC, Ellis RE, Brooks WA. Respiratory patterns associated with swallowing: Part 2. Neurologically impaired dysphagic patients. Age Ageing. 1989;18(3):173-6.

21. Lazareck LJ, Moussavi ZM. Classification of normal and dysphagic swallows by acoustical means. IEEE Trans Biomed Eng. 2004;51(12):2103-12.

22. Boiron M, Rouleau P, Metman EH. Exploration of pharyngeal swallowing by audiosignal recording. Dysphagia. 1997;12(2):86-92.

23. Cook IJ, Dodds WJ, Dantas RO, Kern MK, Massey BT, Shaker R, et al. Timing of videofluoroscopic, manometric events, and bolus transit during the oral and pharyngeal phases of swallowing. Dysphagia. 1989;4(1):8-15.

24. Butler SG, Postma GN, Fischer E. Effects of viscosity, taste, and bolus volume on swallowing apnea duration of normal adults. Otolaryngol Head Neck Surg. 2004;131(6):860-3.

25. Hiss SG, Strauss M, Treole K, Stuart A, Boutilier S. Effects of age, gender, bolus volume, bolus viscosity, and gustation on swallowing apnea onset relative to lingual bolus propulsion onset in normal adults. J Speech Lang Hear Res. 2004;47(3):572-83.

26. Ekberg O, Feinberg MJ. Altered swallowing function in elderly patients without dysphagia: radiologic findings in 56 cases. AJR Am J Roentgenol. 1991;156(6):1181-4.

27. Robbins J, Hamilton JW, Lof GL, Kempster GB. Oropharyngeal swallowing in normal adults of different ages. Gastroenterology. 1992;103(3):823-9.

28. Logemann JA. Evaluation and treatment of swallowing disorders. 2nd ed. Austin: Pro-Ed; 1998

29. Suzuki H, Duprat A, Lederman HM, Bilton T, Tega LP. Comparando avaliação fonoaudiológica, nasolarinscópica e videofluoroscopia em idosos sem queixa de deglutição. Distúrb Comum. 1998; 10(1):91-104.

30. Logemann JA, Pauloski BR, Rademaker AW, Kahrilas PJ. Oropharyngeal swallow in younger and older women: videofluoroscopic analysis. J Speech Lang Hear Res. 2002;45(3):434-45. 
Anexo 1. Roteiro de entrevista inicial

Identificação:

Data:

Nome:

Sexo: ( ) F ( ) M

Idade:

$\begin{array}{lll}\text { Problemas de saúde atuais? ( ) Não ( ) Sim } & \end{array}$

Especificar:

Problemas de saúde anteriores?

( ) Neurológico

( ) Câncer (Cabeça e Pescoço)

( ) Alterações esofágicas/gástricas

Especificar:

Engasgos frequentes?
( ) Não
( ) 1 vez por dia
( ) sólido
( ) 1-2 vezes por mês
( ) todas as refeições
( ) pastoso
( ) Sim
( ) Não

Outras dificuldades de deglutição?

Especificar:

Histórico de disfagia?

( ) Não

( ) Sim

Especificar:

Anexo 2. Questionário para os pais ou responsáveis

Identificação:

Nome da criança:

Sexo: ( ) F ( ) M

Data de Nascimento:
1) Há problemas de saúde atualmente?
( ) Não
( ) Sim

Quais:
2) Problemas de saúde anteriores?
( ) Não
( ) $\operatorname{Sim}$

Quais:

3) Engasgos frequentes?

( ) Não

( ) 1 a 2 vezes por semana

( ) com saliva

( ) com líquido grosso

4) Apresenta outras dificuldades para engolir?

Se sim, explique melhor:

5) Já apresentou em algum momento dificuldade para engolir?

Se sim, explique melhor:

\section{6) Alterações na fala?}

Se sim, explique melhor:

7) Alterações na boca, língua, bochechas, dentes, pescoço, garganta?

Se sim, explique melhor:
Data:

Idade:

( ) de 1 a 2 vezes por mês

( ) 1 vez por dia ( ) todas as refeições

( ) com alimento sólido ( ) com alimento pastoso

( ) com líquido fino
( ) Não
( ) $\operatorname{Sim}$

1- 2 vezes por semana

com saliva

( ) líquido espesso ( ) líquido fino 